\title{
On the N-tuple Wave Solutions of the Korteweg-de Vries Equation
}

By

\author{
Shunichi TANAKA*
}

1. Introduction In this paper, we discuss properties of the $N$-tuple wave solutions of the Korteweg-de Vries (KdV) equation

$$
\dot{u}-6 u u^{\prime}+u^{\prime \prime \prime}=0
$$

where $\dot{u}$ and $u^{\prime}$ are $t$ and $x$ derivatives of $u=u(x, t)(-\infty<x, t<\infty)$ respectively.

The $\mathrm{KdV}$ equation is known to have traveling wave solutions

$$
u(x, t)=s(x-c t-\delta ; c),
$$

where

$$
s(x ; c)=-2^{-1} c \operatorname{sech}^{2}\left(2^{-1} c^{1 / 2} x\right) .
$$

Each of such solutions is called a soliton or solitary wave solution. Recently it was discovered that there exist solutions of the $\mathrm{KdV}$ equation which behave like superposition of two solitons as $t \rightarrow \pm \infty$ (Kruskal and Zabusky [6]). The existence and properties of such solutions (called double wave solutions) were studied by Lax [4].

The structure of the solution of the $\mathrm{KdV}$ equation for the rapidly decreasing initial value was clarified by Gardner, Greene, Kruskal and Miura [2]. They related the solution $u(x, t)$ to the Schrödinger equation with the potential $u$ (for each $t$ ) and found that discrete eigenvalues remain invariant. The reflection coefficient and the normalization coefficients

Received June 10, 1972 and in revised form August 4, 1972.

Communicated by $\mathrm{H}$. Yoshizawa.

* Department of Mathematics, Osaka University, Toyonaka, Japan. 
of eigenfunctions are shown to depend on $t$ exponentially. Here soliton is characterized as the solution with one discrete eigenvalue and the zero reflection coefficient.

According to [2], one calls a solution $N$-tuple wave if for each $t$ corresponding Schrödinger equation has $N$ discrete eigenvalues and its reflection coefficient identically vanishes. As the reflectionless potentials are explicitly constructed by Kay and Moses [3], the possible form of the $N$-tuple wave solution is determined for each $t$. In Section 3 of this paper we give a proof that the function thus constructed is actually a solution of the $\mathrm{KdV}$ equation. Our proof is elementary and independent of the general structure theory of the initial value problem of the $\mathrm{KdV}$ equation such as given by Zakharov and Faddeev [8]. We have to rewrite the formula of the reflectionless potential by Kay and Moses into the form which is more convenient for the study of the $N$-tuple wave (formula (11)). Formula (11) is particularly suitable for the study of the asymptotic behavior of the $N$-tuple wave solution, as each term asymptotically behaves like soliton. This will be discussed in Section 4. Asymptotic behavior of the $N$-tuple wave solutions have been also discussed in Zakharov [7] in a somewhat different form.

After completion of the present paper the author became aware that a result similar to our Theorem in section 3 has been obtained by Hirota [9]. In [9], $N$-tuple wave is given as logarithmic derivative of the determinant of the coefficient matrix of the equation (6) (the formula of Kay and Moses) and the proof has been given by direct calulation. Using the same expression for the $N$-tuple wave, Wadati and Toda [10] have verified that the $N$-tuple wave solutions asymptotically split apart into $N$-solitons.

2. A formula for the reflectionless potential Let $u$ be a realvalued measurable function which satisfies

$$
\int_{-\infty}^{\infty}(1+|x|)|u(x)| d x<\infty
$$


and consider the eigenvalue problem (Schrödinger equation)

$$
-y^{\prime \prime}+u(x) y=k^{2} y
$$

on the infinite interval $(-\infty, \infty)$. For each $k$ with non-negative imaginary part, there exist solutions $g(x, k)$ and $f(x, k)$ of (2) which behave as

$$
\begin{array}{ll}
g(x, k)=e^{-i k x}+o(1) & x \rightarrow-\infty \\
f(x, k)=e^{i k x}+o(1) & x \rightarrow \infty .
\end{array}
$$

For non-zero real $k, f(x, k)$ and $f(x,-k)$ are linearly independent solutions of (2). So $g(x, k)$ can be expressed by them as

$$
g(x, k)=b(k) f(x, k)+a(k) f(x,-k) .
$$

$r(k)=b(k) a(k)^{-1}$ is called the reflection coefficient and $a(k)^{-1}$ is called the transmission coefficient. The eigenvalue problem (2) is known to have only a finite number of negative eigenvalues $-\kappa_{1}^{2}, \cdots,-\kappa_{N}^{2}$ which are simple and corresponding eigenfunctions are given by $f\left(x, i \kappa_{n}\right)$. Put

$$
c_{n}^{-1}=\int_{-\infty}^{\infty}\left|f\left(x, i \kappa_{n}\right)\right|^{2} d x .
$$

$\left\{r(k), \kappa_{n}, c_{n}\right\}$ is called the scattering data of the eigenvalue problem (2) and one can reconstruct the potential $u$ from the scattering data solving the Gelfand-Levitan equation (see Faddeev [1] and Kay, Moses [3]).

If the reflection coefficient is identically zero, the potential $u$ is more explicitly written by the scattering data as follows. Let $\kappa_{1}, \cdots, \kappa_{N}$ be positive numbers different from each other and $c_{1}, \cdots, c_{N}$ be any positive numbers. Put

$$
F(x)=\sum_{n=1}^{N} c_{n} \exp \left(-\kappa_{n} x\right)
$$

and let $K(x, y)(y \geqq x)$ be the solution of the Gelfand-Levitan equation

$$
K(x, y)+F(x+y)+\int_{x}^{\infty} K(x, t) F(t+y) d t=0 .
$$

Putting (4) into (5), we see that $K(x, y)$ has the form 


$$
K(x, y)=\sum_{n} f_{n}(x) \exp \left(-\kappa_{n} y\right)
$$

(we shall hereafter assume that the summation is taken over $1,2, \cdots, N$ unless explicitly indicated). Putting this expression into (5), we have a system of linear equations for $f_{n}(x)$ :

(6) $f_{m}(x)+c_{m} \exp \left(-\kappa_{m} x\right) \sum_{n}\left(\kappa_{m}+\kappa_{n}\right)^{-1} \exp \left(-\kappa_{n} x\right) f_{n}(x)+c_{m} \exp \left(-\kappa_{m} x\right)=0$

$$
m=1, \cdots, N
$$

whose coefficient matrix is easily seen to be invertible. Then the potential $u(x)$ is given by the formula

$$
u(x)=-2(d / d x)\left(\sum_{n} f_{n}(x) \exp \left(-\kappa_{n} x\right)\right)
$$

and $f_{n}$ satisfies the equation

$$
-f_{n}^{\prime \prime}+u f_{n}=-\kappa_{n}^{2} f_{n}
$$

Now let us rewrite the formula (7) into the form which is more convenient for the application to the $\mathrm{KdV}$ equation. Put

$$
h_{n}(x)=f_{n}(x) \exp \left(-\kappa_{n} x\right) \text {. }
$$

Then the equation (6) is written as

$$
\sum_{n} a_{m n}(x) h_{n}(x)=-1
$$

where

$$
\begin{aligned}
& a_{m m}(x)=\left(2 \kappa_{m}\right)^{-1}+c_{m}^{-1} \exp \left(2 \kappa_{m} x\right) \\
& a_{m n}(x)=\left(\kappa_{m}+\kappa_{n}\right)^{-1} \quad m \neq n .
\end{aligned}
$$

Let $\left(b_{m n}\right)$ be the inverse matrix of $\left(a_{m n}\right)$. Then

$$
h_{m}(x)=-\sum_{n} b_{m n}(x)
$$

Differentiation of (9) with respect to $x$ leads to the equation for $h_{n}^{\prime}(x)$ :

$$
\sum_{n} a_{m n}(x) h_{n}^{\prime}(x)=-2 \kappa_{m} c_{m}^{-1} \exp \left(2 \kappa_{m} x\right) h_{m}(x)
$$


So we have

$$
h_{m}^{\prime}(x)=-2 \sum_{n} b_{m n}(x) \kappa_{n} c_{n}^{-1} \exp \left(2 \kappa_{n} x\right) h_{n}(x)
$$

and

$$
\begin{aligned}
u(x) & =-2 \sum_{m} h_{m}^{\prime}(x) \\
& =4 \sum_{m, n} b_{m n}(x) \kappa_{n} c_{n}^{-1} \exp \left(2 \kappa_{n} x\right) h_{n}(x)
\end{aligned}
$$

As the matrix $\left(b_{m n}\right)$ is symmetric, we have finally

$$
\begin{aligned}
u(x) & =-4 \sum_{n} \kappa_{n} c_{n}^{-1} \exp \left(2 \kappa_{n} x\right) h_{n}^{2}(x) \\
& =-4 \sum_{n} \kappa_{n} c_{n}^{-1} f_{n}^{2}(x) .
\end{aligned}
$$

3. N-tuple wave solution Let $u(x, t)$ be a solution of the $\mathrm{KdV}$ equation and consider the Schrödinger equation (2) with the potential $u(x, t)$ for each $t$. Assume that the corresponding reflection coefficient identically vanishes and there exist $N$ eigenvalues $-\kappa_{1}^{2}, \cdots,-\kappa_{N}^{2}$. Time dependency of the normalization coefficient $c_{n}$ is determined in [2] as

$$
c_{n}(t)=c_{n}(0) \exp \left(8 \kappa_{n}^{3} t\right) \quad n=1, \cdots, N
$$

(see also Lax [5]). Conversely we have

Theorem. Let $\kappa_{1}, \ldots, \kappa_{N}$ be positive numbers different from each other and $c_{n}(t)$ be defined by (12). Let $u(x, t)$ be the reflectionless potential which corresponds to the scattering data $\left\{\kappa_{n}, c_{n}(t)\right\}$ for each $t$. Then $u(x, t)$ is a solution of the $K d V$ equation.

Proof. We differentiate (9) with respect to $t$ and obtain equations for $\dot{h}_{n}(x, t)$ :

$$
\sum_{n} a_{m n}(x, t) \dot{h}_{n}(x, t)=8 \kappa_{m}^{3} c_{m}^{-1}(t) \exp \left(2 \kappa_{m} x\right) h_{m}(x, t)
$$

So we have

$$
\dot{h}_{m}(x, t)=8 \sum_{n} b_{m n}(x, t) \kappa_{n}^{3} c_{n}^{-1}(t) \exp \left(2 \kappa_{n} x\right) h_{n}(x, t)
$$




$$
\begin{aligned}
\sum_{m} \dot{h}_{m}(x, t) & =-8 \sum_{n} \kappa_{n}^{3} c_{n}^{-1}(t) \exp \left(2 \kappa_{n} x\right) h_{n}^{2}(x, t) \\
& =-8 \sum_{n} \kappa_{n}^{3} c_{n}^{-1}(t) f_{n}^{2}(x, t) .
\end{aligned}
$$

By (7) we have a formula for the $t$-derivative of $u(x, t)$ :

$$
\dot{u}(x, t)=32 \sum_{n} \kappa_{n}^{3} c_{n}^{-1}(t) f_{n}^{\prime} f_{n}(x, t) .
$$

On the other hand, successive $x$-differentiations of (11) lead to

$$
\begin{aligned}
& u^{\prime}=-8 \sum_{n} \kappa_{n} c_{n}^{-1} f_{n}^{\prime} f_{n} \\
& u^{\prime \prime}=2 u^{2}-8 \sum_{n} \kappa_{n} c_{n}^{-1}\left(\kappa_{n}^{2} f_{n}^{2}+f_{n}^{\prime 2}\right) \\
& u^{\prime \prime \prime}=6 u u^{\prime}-32 \sum_{n} \kappa_{n}^{3} c_{n}^{-1} f_{n}^{\prime} f_{n}
\end{aligned}
$$

where the formula (8) is used. So $u$ satisfies the $\mathrm{KdV}$ equation.

Q.E.D.

4. Asymptotic behavior of the $\mathbf{N}$-tuple wave solutions For $N=1$, the solution of the $\mathrm{KdV}$ equation constructed above takes the form

$$
u(x, t)=-4 \kappa c^{-1} \exp (2 \kappa z) /\left((2 \kappa)^{-1}+c^{-1} \exp (2 \kappa z)\right)^{2}
$$

where $z=x-4 \kappa^{2} t$ and $c=c(0)$. This solution coincides with the soliton

$$
u(x, t)=s\left(x-4 \kappa^{2} t-\delta, 4 \kappa^{2}\right),
$$

where

$$
\delta=(2 \kappa)^{-1} \log c(2 \kappa)^{-1}
$$

We now proceed to the study of the asymptotic property of the general $N$-tuple wave solutions. Put $z_{n}=x-4 \kappa_{n}^{2} t, c_{n}=c_{n}(0)$ and

$$
u_{n}(x, t)=-4 \kappa_{n} c_{n}^{-1} \exp \left(2 \kappa_{n} z_{n}\right) h_{n}^{2}(x, t)
$$

Then each $u_{n}(x, t)$ behaves asymptotically like a soliton. More precisely, suppose $\kappa_{1}<\kappa_{2}<\cdots \cdots<\kappa_{N}$. Then we have 
Proposition. As $t \rightarrow \pm \infty$,

$$
u_{n}(x, t)-s\left(x-4 \kappa_{n}^{2} t-\delta_{n}^{ \pm}, 4 \kappa_{n}^{2}\right) \rightarrow 0
$$

uniformly in $x$, where

$$
\begin{aligned}
& \delta_{n}^{+}=\left(2 \kappa_{n}\right)^{-1} \log \left\{c_{n}\left(2 \kappa_{n}\right)^{-1} \Pi_{i=n+1}^{N}\left(\kappa_{n}-\kappa_{i}\right)^{2}\left(\kappa_{n}+\kappa_{i}\right)^{-2}\right\} \\
& \delta_{n}^{-}=\left(2 \kappa_{n}\right)^{-1} \log \left\{c_{n}\left(2 \kappa_{n}\right)^{-1} \Pi_{i=1}^{n-1}\left(\kappa_{n}-\kappa_{i}\right)^{2}\left(\kappa_{n}+\kappa_{i}\right)^{-2}\right\}
\end{aligned}
$$

Proof. We express $h_{n}(x, t)$ by the Cramer's formula as

$$
h_{n}(x, t)=A_{n}(x, t) A(x, t)^{-1} .
$$

Note that

$$
A(x, t)=\operatorname{det}\left(a_{m n}(x, t)\right)
$$

is a polynomial in $\exp \left(2 \kappa_{1} z_{1}\right), \ldots, \exp \left(2 \kappa_{N} z_{N}\right)$ with positive coefficients and non-zero constant term (Lemma 2 of [3]). It is easy to see that the estimate

$$
\left|h_{n}(x, t)\right|<C\left(1+\exp \left(2 \kappa_{n} z_{n}\right)\right)^{-1} \quad t>0
$$

holds. Therefore $u_{n}(x, t)$ converges to zero as $t \rightarrow \infty$ uniformly in the half spaces $x<\left(4 \kappa_{n}^{2}-\varepsilon\right) t+\delta$ and $x>\left(4 \kappa_{n}^{2}+\varepsilon\right) t+\delta, \delta$ and $\varepsilon$ being any fixed real number and small positive number respectively.

Now we consider the behavior of $h_{n}(x, t)$ in the infinite sector

$$
\left(4 \kappa_{n}^{2}-\varepsilon\right) t+\delta<x<\left(4 \kappa_{n}^{2}+\varepsilon^{\prime}\right) t+\delta^{\prime} \quad t>0 .
$$

We express $A(x, t)$ and $A_{n}(x, t)$ as

$$
A(x, t)=\left(\Pi_{i=1}^{n-1} c_{i}^{-1} \exp \left(2 \kappa_{i} z_{i}\right)\right) B_{n}(x, t)\left(1+R_{n}(x, t)\right)
$$

and

$$
A_{n}(x, t)=\left(\Pi_{i=1}^{n-1} c_{i}^{-1} \exp \left(2 \kappa_{i} z_{i}\right)\right) B_{n}\left(1+Q_{n}(x, t)\right),
$$

where 


$$
\begin{aligned}
& B_{n}(x, t) \\
& =\left|\begin{array}{ccc}
c_{n}^{-1} \exp \left(2 \kappa_{n} z_{n}\right)+\left(2 \kappa_{n}\right)^{-1} & \left(\kappa_{n}+\kappa_{n+1}\right)^{-1} \cdots\left(\kappa_{n}+\kappa_{N}\right)^{-1} \\
\left(\kappa_{n+1}+\kappa_{n}\right)^{-1} & \left(2 \kappa_{n+1}\right)^{-1} & \cdots\left(\kappa_{n+1}+\kappa_{N}\right)^{-1} \\
\vdots & \vdots & \vdots \\
\left(\kappa_{N}+\kappa_{n}\right)^{-1} & \left(\kappa_{N}+\kappa_{n+1}\right)^{-1} \cdots\left(2 \kappa_{N}\right)^{-1}
\end{array}\right|
\end{aligned}
$$

and $B_{n}$ is the determinant obtained by replacing the first column of the above determinant by ${ }^{t}(-1,-1, \ldots,-1)$. Then we have the estimate

$$
\left|R_{n}(x, t)\right|<C\left(\sum_{i=1}^{n-1} \exp \left(-2 \kappa_{i} z_{i}\right)+\sum_{i=n+1}^{N} \exp \left(2 \kappa_{i} z_{i}\right)\right)
$$

in the sector. The same estimate holds for $Q_{n}(x, t)$.

For positive numbers $\alpha_{1}, \ldots, \alpha_{n}$, put

$$
\begin{array}{ll}
D\left(\alpha_{1}, \cdots, \alpha_{n}\right)=\operatorname{det}\left(\alpha_{i j}\right), & \alpha_{i j}=\left(\alpha_{i}+\alpha_{j}\right)^{-1} \\
D_{0}\left(\alpha_{1}, \ldots, \alpha_{n}\right)=\operatorname{det}\left(\alpha_{i j}^{\prime}\right), & \alpha_{i j}^{\prime}=\left(\alpha_{i}+\alpha_{j}\right)^{-1} \quad j>1 \\
& \alpha_{i 1}^{\prime}=1
\end{array}
$$

As $t \rightarrow \infty, u_{n}(x, t)$ behaves like

$$
v_{n}(x, t)=\frac{-4 \kappa_{n} c_{n}^{-1} D_{0}\left(\kappa_{n}, \cdots, \kappa_{N}\right)^{2} \exp \left(2 \kappa_{n} z_{n}\right)}{\left(c_{n}^{-1} D\left(\kappa_{n+1}, \cdots, \kappa_{N}\right) \exp \left(2 \kappa_{n} z_{n}\right)+D\left(\kappa_{n}, \cdots, \kappa_{N}\right)\right)^{2}}
$$

It is easy to see that

$$
v_{n}(x, t)=s\left(z_{n}-\delta_{n}^{+}, 4 \kappa_{n}^{2}\right)
$$

by the relations

$$
\begin{aligned}
D_{0}\left(\alpha_{1}, \ldots, \alpha_{n}\right) & =D\left(\alpha_{2}, \ldots, \alpha_{n}\right) \Pi_{i=2}^{n}\left(\alpha_{1}-\alpha_{i}\right)\left(\alpha_{1}+\alpha_{i}\right)^{-1} \\
D\left(\alpha_{1}, \ldots, \alpha_{n}\right) & =D_{0}\left(\alpha_{1}, \ldots, \alpha_{n}\right)\left(2 \alpha_{1}\right)^{-1} \Pi_{i=2}^{n}\left(\alpha_{1}-\alpha_{i}\right)\left(\alpha_{1}+\alpha_{i}\right)^{-1} \\
& =D\left(\alpha_{2}, \ldots, \alpha_{n}\right)\left(2 \alpha_{1}\right)^{-1} \Pi_{i=2}^{n}\left(\alpha_{1}-\alpha_{i}\right)^{2}\left(\alpha_{1}+\alpha_{i}\right)^{-2}
\end{aligned}
$$

Q.E.D.

By Proposition, as $t \rightarrow \pm \infty$ 


$$
u(x, t)-\sum_{n} s\left(x-4 \kappa_{n}^{2} t-\delta_{n}^{ \pm}, 4 \kappa_{n}^{2}\right) \rightarrow 0
$$

uniformly in $x$. We have moreover the formula for the phase shift of each soliton:

$$
\delta_{n}^{+}-\delta_{n}^{-}=\kappa_{n}^{-1} \sum_{m=n+1}^{N} \log \frac{\kappa_{m}-\kappa_{n}}{\kappa_{m}+\kappa_{n}}+\kappa_{n}^{-1} \sum_{m=1}^{n-1} \log \frac{\kappa_{n}+\kappa_{m}}{\kappa_{n}-\kappa_{m}}
$$

\section{References}

[1] Faddeev, L. D., Properties of the $S$-matrix of the one-dimensional Schrödinger equation, Proc. Steklov Inst. Math. 73 (1964), 314-336 (Russian).

[2] Gardner, O.S., J. M. Greene, M. D. Kruskal and R. M. Miura, A method for solving the Korteweg-de Vries equations, Phys. Rev. Letters, 19 (1967), 1095-1097.

[3] Kay, I. and H. E. Moses, Reflectionless transmission through dielectrics and scattering potentials, J. Appl. Phys. 27 (1956), 1503-1508.

[4] Lax, P. D., Integrals of nonlinear equations of evolution and solitary waves, Comm. Pure Appl. Math. 21 (1968) 467-490.

[5] Nonlinear partial differential equations of evolution, Actes Congrès Intern. Math., (1970). 2, 831-840.

[6] Zabusky, N. J. and M. D. Kruskal, Interaction of solitons in a collisionless plasma and the recurrence of initial states, Phys. Rev. Letters, 15 (1966), 240-243.

[7] Zakharov, V. E., Kinetic equation for solitons, Soviet Physics JETP, 33 (1971), 538-541.

[8] Zakharov, V. E. and L. D. Faddeev, Korteweg-de Vries equation-a completely integrable Hamiltonian system, Functional Analysis and Its Applications, 5 (1971), 18-27 (Russian).

[9] Hirota, R., Exact solution of the Korteweg-de Vries equation for multiple collisions of solitons, Phys. Rev. Letters, 18 (1971) 1192-1194.

[10] Wadati, M. and M. Toda, The exact $N$-soliton solution of the Korteweg-de Vries equation, J. Phys. Soc. Japan, 32 (1972) 1403-1411. 
